\section{OBSERVATIONS IN DIPHTHERIA.}

Presented to the Section on Diseases of Children at the Forty-ninth Annual Meeting of the American Medical Association, held at Denver, Colo., June 7-10, 1898.

BY H. D. JEROWITZ, M.D.

$$
\text { KANSAS CITY, MO. }
$$

It is not the purpose of this paper to lecture upon diphtheria and its treatment before this assembly of eminent physicians nor to enter into any detailed bacteriologic study which would perhaps be only an incomplete reiteration of principles thoroughly known, discovered and taught by some of the other members present; but merely to base my remarks upon conclusions drawn from an impartial observation covering an experience of seven years, which, as has been my good fortune, has been much larger than one would naturally expect for that short space of time. As to conclusions, it must be remembered that experiences vary with different observers; depending 1 , upon the virulence of the epidemic, and 2, upon the mode of treatment. A third item may also be mentioned, as it plays an important rôle in deductions made in this article, and that is the susceptibility and resisting powers of certain classes or sects of people.

From this it is evident that unless the observation includes all the different sects and all kinds of epidemics, statistics would not be reliable. Such an extensive experienoe, however, could not be expected from a general practitioner in a town of 150,000 inhabitants, having no children's hospital or any other opportunity of studying the cases, except as they occur in private practice. The observations include several hundred cases of "exudative" or "membranous" angina, having the diphtheria bacillus. The majority of these cases were comparatively mild and some could not be distinguished from an ordinary sore throat with either a punctate or a coalescent exudation. A certain number (about 8 per cent.) were followed by paralysis and some had laryngeal involvement (about 4 per cent.). For a long time I. was reluctant to call these cases diphtheria, as they did not appear serious enough.

It seems that the ancient diphtheria described in text-books and the modern diphtheria, having as its principal diagnostic point the presence of the Klebs. Loeffler bacillus without regard for the clinical appearance, are two different things. The books describe diphtheria as a severe sickness with profound septic poisoning, a depressed heart and a rapid tendency to cardiac failure, the latter being the chief cause of death, and occurring often inside of twentyfour hours. The patient is said to present an anemic appearance and the fever is not high. The membrane upon which they dwell a good deal is said to be gray and within the mucous membrane, not a superficial exudation. It is not detachable, as it is really the mucous membrane itself, whose only possible way of removal is sloughing off, leaving a raw ulcerated surface which has to heal. The glands of the neck are enlarged, which is also considered a constant symptom. The mortality is given all the way from 50 to 100 per cent., and some physicians even today believe that when a case recovers it was not diphtheria.

The modern diphtheria of today is any sore throat. even without exudation, which will give a culture of the Klebs.Loeffler bacillus. The child may appa. rently be well and play; there need be no cervical enlargement, no dirty membrane which is undetachable; the principal thing is the germ. In some of these cases there is a decomposition going on in the throat with septic infection and usually a fatal result. It is evidently the fact that formerly only these septic cases were considered diphtheria; hence, the great rate of mortality. But now, when the diagnosis is made so much oftener, I find that diphtheria is not such a dreadful disease as generally supposed, and whose danger is, like in measles, scarlet fever, etc. principally from the complications.

The most important of these are involvement of the deeper respiratory passages, nephritis with uremic poisoning, septic infection, and sudden cardiac paral ysis. The clinical picture is about as follows: Sud. den onset with high fever, chilliness, and general malaise; sometimes vomiting and occasionally convulsions. Very often the throat is not complained of and a diagnosis can easily be overlooked, until the condition becomes serious. Such cases as these, if resulting fatally, unnecessarily increase the mortality rate; hence it should be the rule of every physician who has been called in to see a sick child to examine the throat, even though he be positive of its useless. ness. On examining the fauces we find a white or yellowish patch on one or both tonsils, extending sometimes to the posterior wall of the pharynx and uvula, and even further. This exudation can in a great many cases be removed, but it will soon reappear. If left alone it will disappear in from ten to fourteen days-sometimes longer. It does not slough off, in some cases it simply loosens and is spit up, while in cases where it is more adherent it seems to become absorbed, getting thinner and thinner until it finally disappears, leaving a healthy mucous surface, which in some cases may appear slightly congested, but not raw.

When the lower air passages become involved, unless the trouble begins there, the attack is usually sudden and entirely distinct from the pharynx. It does not extend from the latter to the larynx, but is a separate procees. It is recognized by the hoarse and croupy cough, and the stridulous respiration.

Uremic symptoms occurred in about 5 per:cent. of the cases, and these would invariably have paralysis. Albumin was rarely found, being absent in many cases having uremic symptoms. The paralysis involved principally the muscles of the throat. In three cases it affected the ciliary muscle of the eye, having the same effect as if a mydriatic had beon employed. In one case it involved the left arm; in one both lower extremities; in two the muscles of the back and the back of the neck, which resulted in a drooping of the head; in one beginning with the muscles of the throat, extending to the extremities, then to the muscles of the chest and finally the heart; and in still another, now under observation, the paralysis involves the sphincters of the bladder and anus and the muscles of the left hip and leg. With the exception of the one fatal case and the one now under observation, the paralysis disappeared, varying in its course from one week to three months.

The number of deaths was seven. This is about $1 \frac{3}{4}$ per cent. A brief description of each would perhaps be of interest.

Case 1.-Female child, age 31\% years. Had been sick for a week before medical aid was received. Pharynx, larynx and trachea involved. Tracheotomy was advised but refused until the patient was almost exhausted. It was then performed but patient died next day from exhaustion. No antitoxin used. Case 2.-Male child, age 31/2 years. Began slightly on tonsils, but child played on the street right along, and soon 
became croupy, and was so for three days before I was called. Finding just a small speck of exudation on one tonsil, and having treated the other children of the family that week for a mild diphtheria, I warned the mother of the seriousness of the case; but she said the child always was croupy whenever he took cold, and disregarded the advice. The next morning the condition became worse, the breathing very stridulous and difficult and the lips cyanotic. After an unsuccessful attempt at intubation, tracheotomy was performed and the child at once improved. The membrane involved the wound and twelve days afterward the child died at night from neglect by allowing the tube to get plugged up. No antitoxin was used.

Case 3.-Female child 31/2 years old. Took suddenly sick with laryngitis one day, and the next day the obstruction was so complete that the child was apparently dead, when intubation was attempted, but as it was not considered safe with this, tracheotomy was resorted to. Then with artificial respiration and application of heat to the body, the child rallied after about five hours and was able to sit up and drink. The fol lowing morning the child died of neglect, the inner tube hav ing been laid on a table by the attendant and forgotten to be replaced, so that when the child was beginning to suffocate, the attendant in trying to remove the inner tube (which was not there) lost so much time by thinking it was caught in some way, that the child succumbed. In this case no membrane was visible and no antitoxin was used.

Cases 4 and 5.-These two cases were one male and one female, age $3 \frac{1}{2}$ years, who had septic infection and laryngeal involvement. Antitoxin was used too late and shortly before death tracheotomy was performed, which relieved the dyspnea for an hour, when they succumbed to cardiac weakness dependent on the septicemia.

Case 6.-Female child 31\% years old. Had, together with the other children of the family, tonsillar diphtheria, which disappeared in about three or four days in all of them. But in this one about three days afterward, fever developed, and a slight patch reappeared on the tonsils. When the child eried there seemed to be a stridulous or sawing noise which led to an examination of the lungs. There were subcrepitant râles showing that the lungs must have been involved. There was no cough nor hoarseness. The child was apparently well, and when asleep there was not the slightest noise with the respirations. Only when the child cried could this peculiar sound be heard. This child lived another week after this, dying from slow carbonic acid poisoning, as was noticed by the gradually increasing cyanotic appearance. No antitoxin was used.

Case 7.-This case was a tonsillar diphtheria of one side. The membrane disappeared in flvedays by gradual absorption: then came uremia and paralysis. The urine was full of albumin, dropsy developed, and the paralysis increased until it in volved almost the entire body. Soon the albumin and dropsy disappeared, the paralysis became somewhat better, the child sat up, and in reaching for something, sudden death occurred. This was about six weeks from the beginning of the illness. No antitoxin had been used.

In summing up these cases, No. 1 might have been saved with antitoxin, if used early enough. No. 2 died of neglect, but antitoxin would have prevented the operation. No. 3 had no visible membrane, and died of neglect. Nos. 4 and 5 could not have been saved. No. 6 could have been saved with antitoxin if it had boen used in the first attack. No. 7 might have been saved by antitoxin, but as the exudation was of short duration, and the general symptoms mild, it is a question.

Thus, out of the seven fatal cases, only three could be really attributed to the disease. This would make a mortality of three-fourths per cent.

\section{TREATMENT.}

As to the treatment, I only wish to mention a few points. It is useless to detach the membrane, for two reasons: First, it quickly reappears, thus giving it new activity and prolonging the disease. Second, by forcibly detaching, the surface is abraded, and septic infection easily follows. I dare say that if you leave the membrane alone in all cases, you will have less septicemia and less deaths. Gargles or sprays should be mild, antiseptic, and often. When not practicable, antiseptics such as listerin, hydrogen peroxid, etc., can be administered internally. Whisky for stimulation. Plenty of water in all cases, and calomel when indicated, especially in uremia. Iron I never employ, as by its astringent and cauterizing properties it can only interfere with natural drainage and healthy excretion. With this treatment, the disease can not be cut short, nor does it prevent its extension. For this we must employ antitoxin.

\section{ANTITOXIN.}

As to the use of this agent, I can give you my observations from an impartial standpoint. The first thing noticed after using antitoxin is a reaction, an immediate effect, something that we do not observe after other remedies unless we give full doses. It is therefore evident that the antitoxin is a powerful agent, and certainly produces some change in the system.

The second thing noticed is that in every case where I administered antitoxin in the proper dose, the membrane disappeared inside of three days. Without its use, while I have seen cases get well in the same length of time, yet the majority of cases lasted from ten to fourteen days. Third, in all cases where $I$ em. ployed antitoxin in the beginning the larynx did not become involved, and uremia did not occur, while in cases treated without antitoxin the larynx became involved frequently, and uremia also followed. Fourth, in cases where the membrane involves the larynx, trachea, and even the bronchial tubes, recovery followed when treated with antitoxin while the same class of cases before the use of antitoxin always died. Fifth, neglected cases, complicated with septicemia and laryngeal involvement, do not recover with any treatment. Sixth, in sévere cases of dyspnea, with occasional cyanosis, the use of antitoxin and emetics has obviated the necessity for tracheotomy. Seventh measles as a complication is no bar to the use of antitoxin. Eighth, in laryngeal cases complicated with uremia or nephritis antitoxin was followed by favorable results, thus proving that uremia and nephritis are not necessarily contra-indications to the use of antitoxin. Ninth, as paralysis was seen to follow only the cases of long duration, those in which antitoxin was administered early were not followed by paralysis. Of those in which it was given on the second or third day it followed in about 8 per cent. of the cases. From this it follows that antitoxin does not prevent paralysis except indirectly by cutting short the disease. Tenth, place of injection, behind scapula. No abscess or other bad result followed.

\section{INTUBATION.}

This has been resorted to in extreme cases. Some cases were rendered worse, and were at once followed by tracheotomy. Some were only slightly improved, and in these the tube was removed and the patient carefully watched, so that tracheotomy could be performed immediately upon indication therefor, but the severe attacks of suffocation were only transient, and surgical intervention became unnecessary.

\section{TRACHEOTOMY.}

This was performed five times, all proving fatal. Two of them died of neglect, two of them had profound septicemia at time of operation, and were only temporarily improved so $\mathrm{fa}_{2}$ as respiration was concerned, and one was already exhausted from suffocation, owing to procrastination on the part of the parents. The operation was always done without an anesthetic. There were a great many other cases that 
seemed to indicate tracheotomy and looked as if they could not live more than an hour or two, yet they recovered without the operation. Therefore I think that cases reoovering from an early tracheotomy would have done so without it. In cases dying from slow carbon dioxid poisoning from membranous bronchitis or membranous pneumonia, I did not perform tracheotomy, as it seemed to me useless.

Let us summarize a few points:

1. The majority of cases are mild, and affect only the tonsils and pharynx.

2. The exudation lasts from one to two weeks. There are exceptions to this.

3. Removal of the membrane is useless, as by denuding the surface septic infection is promoted, and besides, the membrane soon reappears and remains longer than if it had not been meddled with.

4. The involvement of the larynx is always sudden and comes as a new attack, and not by extension of the diphtheritic process.

5. The larynx becomes involved in about 4 per cent. of the cases.

6. Every case complicated with uremia had postdiphtheritic paralysis.

7. Laryngeal involvement is to be most dreaded between the ages of 3 and 5 .

8. Intubation and tracheotomy will become less and less necessary as the value of antitoxin is being recognized.

9. Conclusions as to antitoxin have been given above.

\section{INDICATIONS FOR INTUBATION.}

Presented to the Section on Diseases of Children at the Forty-ninth Annual Meeting of the American Medical Association, held at Denver, Colo., June 7-10, 1898.

BY H. M. McCLANAHAN, M.D. OMAHA, NEB.

The conclusions deduced from a study of the symp. toms of laryngeal diphtheria, as stated in this paper, are the result of personal observations at the bedside. My sole object in presenting them to the distinguished members of this Seotion is to elicit their candid criticisms, that, profiting by their large experience, I may be the better qualified to decide when to act, when to defer and when to desist.

When called into the presence of a little patient suffering from laryngeal diphtheria, the decision of the question whether to intubate or not is a very grave one, and we can only rightly measure our responsibility when we realize that a human life may be sacrificed by an erroneous decision. Aside from the symptoms, our decision will be modified by two circumstances, namely: the previous treatment and the environment of the patient. : Before the discovery of antitoxin, the majority of cases of laryngeal diphtheria became urgent enough to demand intubation for their relief. Under the influence of this remedy 60 per cent. recover without mechanical aid. It will be apparent then that in a given case our decision will be modified by the fact whether antitoxin has been previously administered. We may defer operation in a oase already under the influence of this remedy, whereas in another case presenting identical symptoms we would feel warranted in intubating at once, because twelve to twenty-four hours must elapse hefore amelioration of symptoms can be expected. In L.ospital practice, with a resident physician constantly at hand and competent to operate, surgical interference may be deferred for immediate urgent symptoms, knowing that the relief of the stenosis can be procured in a few moments. In private practice, where a delay of hours might easily occur before the physician could return to the patient, greater caution is necessary. My conclusions are deduced from cases in family practice, and it is from that standpoint that I wish to discuss the subject.

Let us briefly note the symptoms that indicate the necessity for intubation:

The character of the respiration.-Labored inspiration is a constant symptom in all varieties and degrees of laryngeal stenosis: The importance of this symptom depends upon the amount of muscular effort expended in pumping air into the lungs. There is no way of measuring this expenditure of vital force more certainly and accurately than by observing the extent of the epigastric recession with each inspiration. Where there is noticed a deep depression, we may be certain that the patient is in a critical condition, and we have no assurance as to how long he may be able to continue this effort. Intubation here meets two important indications: More oxygen is received into the lungs, and violent muscular effort is sus. pended, increasing the vitality and saving the strength of the patient. Labored expiration is an important symptom; to my mind, the most important evidence of a pseudo membrane in the larynx. While this is usually associated with labored inspiration and other symptoms, the two are not always in proportion; for example, in one case each expiratory effort was prolonged and labored, with a pause at the end of the inspiratory act, but inspiration was comparatively easy, with but slight epigastric recession, yet this patient was both cyanotic and extremely restless. Intubation restored the rbythm of the respiratory act with prompt relief of the cyanosis.

The number of respirations per minute.-This is important, as indicating the extent of involvement of the respiratory tract. In laryngeal diphtheria my observation has been that the respirations are not greatly accelerated, whereas if there be involvement of the lesser tubes and air vesicles, we find both labored and rapid respirations. This class of cases present the most unfavorable prognosis, not so much on account of the laryngeal stenosis as of the septic pneumonia complicating the case. While in these cases intubation will relieve the labored respiratory act, it does not lessen the frequency of the respirations. I believe, however, the operation is indicated, not only to relieve the stenosis, but to enable the child to cough more vigorously, and more effectually to clear out the lesser bronchial tubes. In two cases where the symptoms were not extremely urgent there were attacks of dyspnea, due to spasm of the muscles of the larynx, presenting a typic picture of spasmodic croup. Both became markedly cyanotic during the attack. There was no evidence that this was due to particles of loosened membrane, and I am satisfied from the study of the cases that it was a spasmodic condition complicating the true croup. This complication adds greatly to the suffering of the patient and the anxiety of the friends. The history of such attacks, coming on at irregular intervals, in a case where the other symptoms are not extremely urgent in the interim, in my experience, demands intubation. In the two cases of this type-while I do not affirm that they would have terminated fatally-I do 